\title{
Editorial \\ NPP: Our Designs for the Future
}

\author{
William A Carlezon Jr, I and Anissa Abi-Dargham² \\ 'Department of Psychiatry, Harvard Medical School, Belmont, MA, USA; ²Department of Psychiatry, Stony Brook School of Medicine, Stony Brook, \\ NY, USA
}

Neuropsychopharmacology (2017) 42, I37|-1372. doi: I 0. I 038/npp.20 I7.54

Neuropsychopharmacology (NPP) has now been published by Springer Nature (SN), or its previous incarnation (Nature Publishing Group, NPG), for 15 years. The American College of Neuropsychopharmacology (ACNP) recently renewed our contract with $\mathrm{SN}$, reaching an agreement that covers the next 10 -year period. The new contract brings changes and updates to NPP, including the redesigned cover that adorns this issue. It also serves as an occasion to recall important points in the history of the journal, highlight recent changes, and articulate future plans.

NPG took over as publisher of NPP in 2002. At the time, journals were evolving from paper-based systems-where authors submitted manuscripts as hardcopies and journals communicated with reviewers by faxes and overnight couriers -to online review platforms. To modernize the look of the printed journal, a so-called 'living cover' (Figure 1) was designed; the key element being that each month includes a new image that moves across the cover (right to left) over the course of a 3-month cycle. This design is unique and has become a signature feature of NPP, simplifying the decision to retain it in the cover redesign. In 2008, the popular ACNP 'Generation of Progress' textbook series was discontinued and replaced by Neuropsychopharmacology Reviews, a themebased collection of review articles published each January as a special issue of NPP that has become a major driver of our impact factor (IF). In 2013, the tagline 'At the intersection of brain, behavior, and therapeutics' was added to the cover to sharpen our brand, distinguish us from similarly named journals, and indicate that our interests extend beyond the traditional pharmacology.

Publishing has changed considerably since 2002. Most noticeably, there are now many more journals and a higher percentage that publish open access (OA) only. Each day brings a relentless flow of invitations from new journals to serve as a reviewer, or offers to publish our research. Not all of these journals are reputable, and some take advantage of the OA model to set up fee-for-publication schemes, an alarming development at a time when funding bodies like the National Institutes of Health (NIH) are prioritizing rigor and

*Correspondence: Dr WA Carlezon Jr, Department of Psychiatry, McLean Hospital, MRC 217, I 5 Mill Street, Belmont, MA 02478, USA, Tel: 617855 2021, Fax: 617855 2023,

E-mail: bcarlezon@mclean.harvard.edu reproducibility. Characteristic in these schemes is a peerreview process that is either suspiciously expedited or totally absent. While NPP offers an OA option, which entails an article processing charge because it makes the final version of papers free to all readers immediately regardless of subscription, our standards for publishing are higher (more selective) than ever. We are actively introducing new features designed to make the journal maximally interesting and informative, as well as helpful in times of word, page, and citation limits. One such feature is Circumspectives, a debatelike forum that ends with a co-authored section that describes what is needed to move the field forward. This article type is designed to represent 'one-stop shopping' for researchers: these are single papers that describe the full scope of an issue and include the seminal citations, which is especially helpful for authors when space and/or word limits require brevity. Circumspectives is having a major impact: our first (Wise and Koob, 2014) is currently the most highly cited paper of that year, and our most recent (Bale and Epperson, 2017) provides a comprehensive vision of how sex difference studies should be approached in response to new NIH guidelines. Our review and production processes are consistently among the fastest in the field, and beginning within $48-72 \mathrm{~h}$ of acceptance, new papers are online (and citable) in their most updated formats via the accepted article preview and advance online publication features. We have added more commissioned review articles, commentaries, perspectives, and research highlights. Recognizing that researchers no longer read hardcopy issues from cover to cover, but rather obtain individual articles when they need them from via online library collections, we have established journal social media accounts that highlight new content, and can nimbly call attention to older content in a way that is maximally responsive to news or developments in the field. Finally, we have added our nickname (NPP) as a watermark to our redesigned cover, to boost familiarity, and create an added sense of connection between the journal and our readers.

For the foreseeable future, we will be prioritizing work that provides insights on the mechanisms of psychiatric illness and its treatment. We seek to publish a mixture of clinical and preclinical (basic neuroscience) research, mirroring the missions of the ACNP and its membership. Currently, our foremost goal is to attract more high-quality clinical papers. 


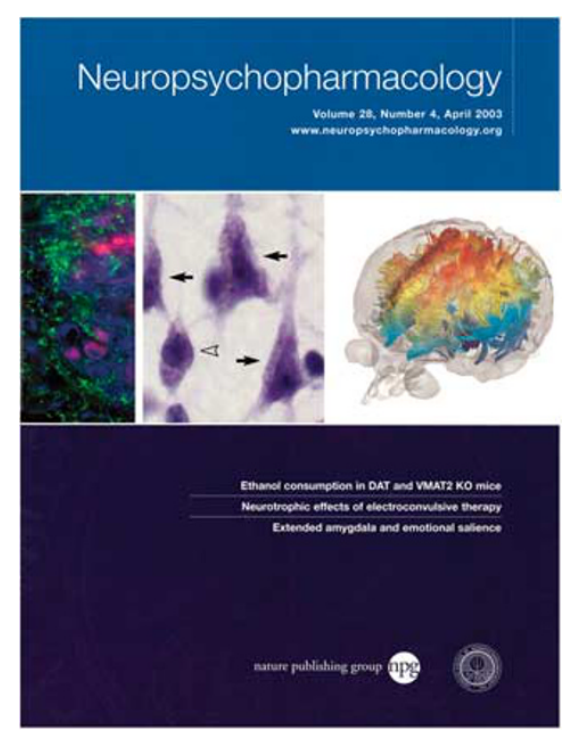

Figure I An early example of the 'living cover', from the first volume of NPP published by NPG in 2003. With the exception of some minor modifications (addition of a tagline in 2013), the cover design remained unchanged for almost 15 years.

This includes all types of psychiatry-related research involving humans-not just clinical trials. Over the course of the next year, we intend to introduce features and article types that will represent exciting new forums for clinical research and care. For preclinical work, we wish to attract original research that is innovative and thorough, and geared to have an impact on our understanding of brain function in the context of treating or preventing psychiatric illness. We prioritize mechanistic studies but, in general, believe that the quality of a discovery rather than a 'wall of data' (ie, figures with dozens of embedded sub-figures) is the most important criterion for publication. We recognize that molecular biology has driven most recent technical advances in the field, and that this has created striking mismatches between the sophistication of the methods used to manipulate brain function and those used to evaluate behavioral outcomes. The development of methods that enable collection of analogous end points in laboratory animals and humans is important for the future of translational research, and we will be seeking papers that innovate in this domain. True to our tagline-and recognizing that 'brains have owners' (Yong, 2017) - we want to be a journal where technical innovation can be molecular, behavioral, or (ideally) both.

In the year ahead, we will be rolling out developments that will further enrich the reader's experience with online content. We will continue to emphasize the availability of new tools (eg, ReadCube) and metrics (eg, Altmetrics) that enable real-time insight on how frequently papers are being viewed and the demographics of those who are viewing them. This moves us toward a system where the true impact of an individual paper can be more accurately judged, as opposed to the present system whereby papers take on the IF of the journal in which they are published, regardless of whether the work is cited or widely reproducible.

The renewed partnership with SN strengthens our position as an asset for the ACNP and our ability to serve as a nexus for all fields operating within the domain of neuropsychopharmacology. We hope that NPP enhances your ability to conduct innovative research and/or provide innovative care, and that you continue to consider us as a forum for your best work.

\section{FUNDING AND DISCLOSURE}

WAC is the current Editor-in-Chief of NPP and AA-D is the current President of ACNP. The authors declare no conflict of interest.

\section{REFERENCES}

Bale TL, Epperson CN (2017). Sex as a biological variable: who, what, when, why, and how. Neuropsychopharmacology 42: 386-396.

Wise RA, Koob GF (2014). The development and maintenance of drug addiction. Neuropsychopharmacology 39: 254-262.

Yong E (2017). How Brain Scientists Forgot that Brains have Owners (The Atlantic), 27 February, https://www.theatlantic.com/ science/archive/2017/02/how-brain-scientists-forgot-that-brainshave-owners/517599/. 